\title{
The imperative to reduce carbon emissions in astronomy
}

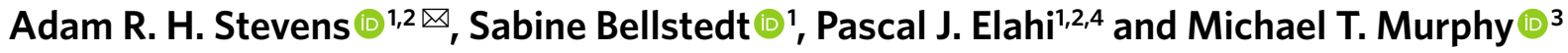

For astronomers to make a significant contribution to the reduction of climate change-inducing greenhouse gas emissions, we first must quantify the sources of our emissions and review the most effective approaches for reducing them. Here we estimate that Australian astronomers' total greenhouse gas emissions from their regular work activities are $\gtrsim \mathbf{2 5}$ ktCO$_{2} \mathrm{e} \mathrm{yr-1}(\mathrm{equivalent}$

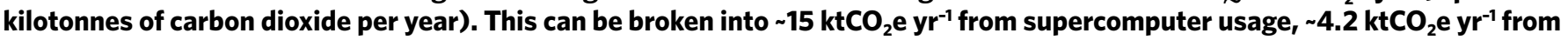
flights (where individuals' flight emissions correlate with seniority), $>3.3 \mathrm{ktCO}_{2} \mathrm{e} \mathrm{yr}^{-1}$ from the operation of observatories, and $2.6 \pm 0.4 \mathrm{ktCO}_{2} \mathrm{e} \mathrm{yr}^{-1}$ from powering office buildings. Split across faculty scientists, postdoctoral researchers and PhD students, this averages to $\gtrsim 37 \mathrm{tCO}_{2} \mathrm{e} \mathrm{yr}^{-1}$ per astronomer, more than $40 \%$ greater than the average Australian non-dependant's emissions in total, and equivalent to around five times the global average. To combat these environmentally unsustainable practices, we suggest that astronomers should strongly preference the use of supercomputers, observatories and office spaces that are predominantly powered by renewable energy sources. Where current facilities do not meet this requirement, their funders should be lobbied to invest in renewables, such as solar or wind farms. Air travel should also be reduced wherever possible, replaced primarily by video conferencing, which should also promote inclusivity.

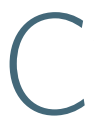
limate change is widely regarded as the biggest ongoing issue facing the planet's inhabitants right now. So much so that over 11,000 scientists from 153 countries recently signed a paper warning of a global climate emergency ${ }^{1}$. Humanity's continuing emission of greenhouse gases-driven predominantly by the burning of fossil fuels as a source of energy ${ }^{2}$ - has already led to a rise in the mean global surface temperature of $\sim 1{ }^{\circ} \mathrm{C}$ relative to pre-industrial levels ${ }^{3}$. For global heating to be limited to $1.5-2{ }^{\circ} \mathrm{C}$ as per the Paris Agreement requires a decrease to effectively zero anthropogenic emissions in the next few decades ${ }^{4-7}$. Even then, it is expected that there will be long-lasting (timescales of $\gtrsim 10^{3}-10^{5} \mathrm{yr}$ ) or potentially permanent changes to the environment ${ }^{8-10}$, which will have (and are already having) widespread, substantial impacts on many forms of life. This has been discussed in the literature and media for decades, with a complete technical elaboration provided as part of the Fifth Assessment Report from The United Nations' Intergovernmental Panel on Climate Change ${ }^{11}$.

As is the case for most (if not all) professions, there are many aspects to being an astronomer that currently result in the emission of greenhouse gases and, therefore, a direct contribution to climate change. Broadly, these include direct emissions from flights, and indirect emissions from the electricity required to power supercomputers, observatories and other facilities, in addition to emissions associated with their construction. We are no less responsible for ensuring we reduce our emissions from these activities than anyone else in the world is for reducing their own sources of emissions.

To address methods for emissions reduction demands that one understands not only where their own sources of emissions come from, but also what their relative quantitative significance is. Part of the purpose of this Perspective is to provide astronomers with a base level of quantitative information on their sources of emissions. More than this though, it is imperative that acknowledgement of this leads to action that will result in a decrease in the community's emissions. For to be aware of a problem but choose not to act is practically no different than to deny the problem's existence, especially when one is demonstrably contributing to said problem ${ }^{12,13}$. We all have an ethical obligation here that must not be ignored.

Climate change action is particularly important for Australiabased astronomers (and Australians in general), as Australia's record of greenhouse gas emissions is particularly poor in the global context. Australia's total emissions (excluding international flights and shipping) for the year ending March 2019 were 538.9 million equivalent tonnes of $\mathrm{CO}_{2}\left(\mathrm{MtCO}_{2} \mathrm{e}\right)\left(\right.$ ref. $\left.{ }^{14}\right)$. With a population of 25.287 million people at the end of the March 2019 quarter according to the Australian Bureau of Statistics—of which 18.7\% are dependants under the age of 15-the country's emissions rate equates to 26.2 $\mathrm{tCO}_{2} \mathrm{e} \mathrm{yr}^{-1}$ per non-dependant. This is in stark contrast to the 2018 global average of $7.3 \pm 0.7 \mathrm{tCO}_{2} \mathrm{e} \mathrm{yr}^{-1}$ per non-dependant (based on total emissions from the Global Carbon Budget $2019^{2}$ and the global population from Worldometer, taking half the range of the 2017 and 2019 values as the uncertainty on the latter) and makes Australia one of the highest-emitting countries per person in the world. Countries that have comparable per-capita emission rates to Australia include the United States and Canada ${ }^{15}$. Perhaps it is no coincidence then that members of the astronomical communities from these countries have written white papers on this same topic, which include several practical, sensible suggestions for mitigation strategies ${ }^{16,17}$. This is clearly an issue that astronomers worldwide are cognisant of; the Canadian paper $^{16}$ was one of the five most widely discussed papers for its month of release, with members from 43 astronomy institutes up-voting it on the Voxcharta website. In Australia, an open letter has been written to the federal government, highlighting the urgent need to reduce greenhouse gas emissions, which has been signed by over 80 Laureate Fellows-the most

IInternational Centre for Radio Astronomy Research, The University of Western Australia, Crawley, Western Australia, Australia. ${ }^{2}$ Australian Research Council Centre of Excellence for All Sky Astrophysics in 3 Dimensions (ASTRO 3D). ${ }^{3}$ Centre for Astrophysics and Supercomputing, Swinburne University of Technology, Hawthorn, Victoria, Australia. ${ }^{4}$ Present address: Pawsey Supercomputing Centre, Kensington, Western Australia, Australia. 凶e-mail:adam.stevens@uwa.edu.au 
Table 1 | Typical emissions for (the most) direct return flights between Australian capital cities, according to Qantas

\begin{tabular}{lllllll} 
& ADL & BNE & CBR & HBA & MEL & PER \\
\hline BNE & 340 & & & & & \\
CBR & 240 & 306 & & & & \\
HBA & 378 & 314 & 296 & & & \\
MEL & 134 & 288 & 122 & 144 & & \\
PER & 442 & 748 & 674 & 656 & 528 & \\
SYD & 246 & 158 & 82 & 236 & 148 & 652 \\
\hline
\end{tabular}

Three-letter names are the official airport codes. Units for emissions are $\mathrm{kgCO}_{2} \mathrm{e}$ per passenger (rounded to the nearest integer).

senior and prestigious research fellows funded by the Australian Research Council-including six astronomers.

In this Perspective, we take approximate stock of the greenhouse gas emissions for which Australian astronomers are responsible (see the 'Sources of astronomers' emissions' section). We then present options for how these sources may (and should) be reduced, and discuss current initiatives along these lines (see the 'Solutions and discussions' section). Our focus on the Australian community is a practical one, as it reflects the fact that we-the authors-are all based in Australia. Despite this, the underlying content and message of this paper should be relevant for the global astronomical community and other fields of science.

\section{Sources of astronomers' emissions}

In this section, we provide an overview of the emissions that Australian astronomers are responsible for, from the sources of expected greatest significance, in no specific order.

Flights. Relative to the general public, astronomers travel a lot. Reasons include, but are not limited to: conferences, workshops, collaboration, seminars, observing runs, committee meetings, job interviews, and relocation. This is not specific to astronomers though; academics in general are responsible for considerable greenhouse gas emissions due to flying. One case study suggests that business-related flights from university employees contribute approximately two thirds of the emissions of campus operations ${ }^{18}$. Flights are often the greatest single source of university emissions, with conference attendance accounting for approximately half of those flight emissions ${ }^{19}$.

Not only does all international travel require flying thousands of kilometres from Australia, but due to the size and low population density of the country, domestic travel often does too. As a point of reference, we collate the approximate greenhouse gas emissions per passenger from direct flights between Australian capital cities in Table 1, according to Qantas. Based on the same carbon calculator, return trips from Australia to Europe or the Americas can comfortably exceed $3 \mathrm{tCO}_{2} \mathrm{e}$ per passenger.

In Australia, aviation was responsible for $22.02 \mathrm{MtCO}_{2} \mathrm{e}$ of emissions in 2016 alone (which includes $12.02 \mathrm{MtCO}_{2}$ e from international flights ${ }^{20}$. This suggests that aviation is responsible for $\sim 4 \%$

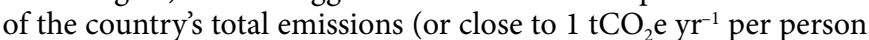
on average). While this may sound like a small fraction, it is important to recognize that about half the population will not fly at all in a given year, that most of them will only fly once in that year, and that the vast majority will do so for leisure, not business. For the relatively few people who fly regularly, their personal fraction of emissions from air travel presumably must be much higher than the nominal $4 \%$. As we demonstrate in this section, astronomers are among those people (certainly, at least, in Australia).
CAS budget example. As an example of astronomy's disproportionately high flight emissions, consider Swinburne University of Technology's Centre for Astrophysics and Supercomputing (CAS). In 2017, approximately $80 \%$ of CAS's travel budget was spent on flights: $\sim \mathrm{AU} \$ 301,000$ in total (including external funding contributions), with AU $\$ 54,000$ spent on 134 domestic round-trip flights, and the remaining AU $\$ 247,000$ spent on 133 international round trips (often including more than two flights). These flights covered the $\sim 80$ full-time-equivalent (FTE) staff and students in CAS during 2017, meaning each person was responsible for $\sim 1.7$ domestic and $\sim 1.7$ international flights on average. A typical domestic return flight from Melbourne produces $\sim 230 \mathrm{kgCO}_{2}$ e per passenger (taking a naïve average of the values for MEL in Table 1). Considering Los Angeles as a typical international destination, a return international flight produces $\gtrsim 3 \mathrm{tCO}_{2} \mathrm{e}$ per passenger (from Qantas's calculator). Therefore, the average astronomer in CAS was responsible for $\sim 5.4$ $\mathrm{tCO}_{2} \mathrm{e}$ in 2017 from flying alone, with 0.4 and $5.0 \mathrm{tCO}_{2} \mathrm{e}$ coming from domestic and international flights, respectively. As a rough guide to the average monetary carbon cost of flying, these figures imply $\sim 0.57 \mathrm{kgCO}_{2} \mathrm{e}$ per AU\$ for domestic flights and $1.6 \mathrm{kgCO}_{2} \mathrm{e}$ per AU\$ for international flights. These figures are comparable to the case study of Stohl ${ }^{21}$ at a different institute (and research field).

ICRAR-UWA travel records. A further, more detailed example is available from the International Centre for Radio Astronomy Research-University of Western Australia node (ICRAR-UWA). Here, the complete travel records for the 2018 and 2019 calendar years were analysed. Over this time, ICRAR-UWA used three different travel agencies. All work-related travel captured by these agencies was accounted for, regardless of the funding source. Two of those agencies gave direct emissions values for all bookings captured by their systems. For the third agency, we still had access to all flights travelled, but had to calculate the emissions for each flight; for this, we used Qantas's calculator.

All emissions initially quoted did not differentiate between economy and business class flights. Business class seats occupy roughly triple the area of economy seats (this varies plane to plane, and is often lower for domestic trips and higher for international trips, with one article suggesting a factor of 3.5 is more common for the latter). For the relatively few business class flights listed in the travel records, we multiplied their emissions by three. We emphasize that economy class is the norm for astronomers, and the vast majority of bookings in these records were indeed economy.

In Table 2 and Fig. 1, we summarize the findings from ICRAR-UWA's travel records. While these data have been anonymized, we present statistics for different levels of staff. Where we refer to 'senior scientists', we mean all research staff employed at Level $\mathrm{C}$ and above in the Australian university employment system, which are effectively all tenured or tenure-track positions, including senior fellows, associate professors and full professors. We broadly label all nominal research staff employed at Level A or B as a 'postdoc', all of whom are on fixed-term contracts, which includes research associates and early-career fellows. All remaining staff who are not students fall under the 'professional' category. This covers a diverse range of staff, including outreach, administration, computer scientists and engineers. Masters and $\mathrm{PhD}$ students are considered separately. Other students are not explicitly accounted for (for example, honours students, of which ICRAR-UWA has none).

Unsurprisingly, flight frequency-and thus flight emissionsscales with seniority (as has been found in other studies ${ }^{18}$ ). The average senior staff member emits close to 12 equivalent tonnes of $\mathrm{CO}_{2}$ from flying each year (or roughly four return international trips, or three international plus four domestic). Granted, this mean (but not the percentiles) is pulled up by two outlier points in the distribution; removing the factor-of-three assumption regarding emissions of business versus economy seats would reduce this mean 
Table 2 | Summary of greenhouse gas emissions from ICRAR-UWA employees' work-related flights from 2018 and 2019

\begin{tabular}{|c|c|c|c|c|c|c|}
\hline \multirow[t]{2}{*}{ Staff type } & \multirow[t]{2}{*}{ Number } & \multicolumn{5}{|c|}{$\mathrm{CO}_{2}$ emissions per person $\left(\mathrm{t} \mathrm{yr}^{-1}\right)$} \\
\hline & & 16th percentile & Median & 84th percentile & Mean & Mean including guests \\
\hline Masters students & 16 & 0.00 & 0.00 & 0.00 & $0.21(0)$ & \\
\hline Postdocs & 14 & 0.00 & 2.70 & 7.00 & $3.16(2)$ & \\
\hline Senior scientists & 22 & 3.16 & 8.45 & 17.77 & $11.91(6)$ & $13.87(6)$ \\
\hline Professionals & 20 & 0.00 & 0.00 & 4.67 & $1.76(1)$ & \\
\hline All & 100 & 0.00 & 0.63 & 7.34 & $3.84(0)$ & $4.27(0)$ \\
\hline
\end{tabular}

Emissions are measured in equivalent tonnes of $\mathrm{CO}_{2}$ per person per annum. See the 'ICRAR-UWA travel records' section for a full description of each staff type. The second-to-last row combines results from PhD students, postdocs and senior scientists. The final row does the same, with the additional inclusion of masters students and professional staff. The second mean column (last column) adds the contribution from guest flights that were captured by the ICRAR-UWA booking system. Uncertainties on the last digit for the means are calculated from jackknifing and are included in parentheses.

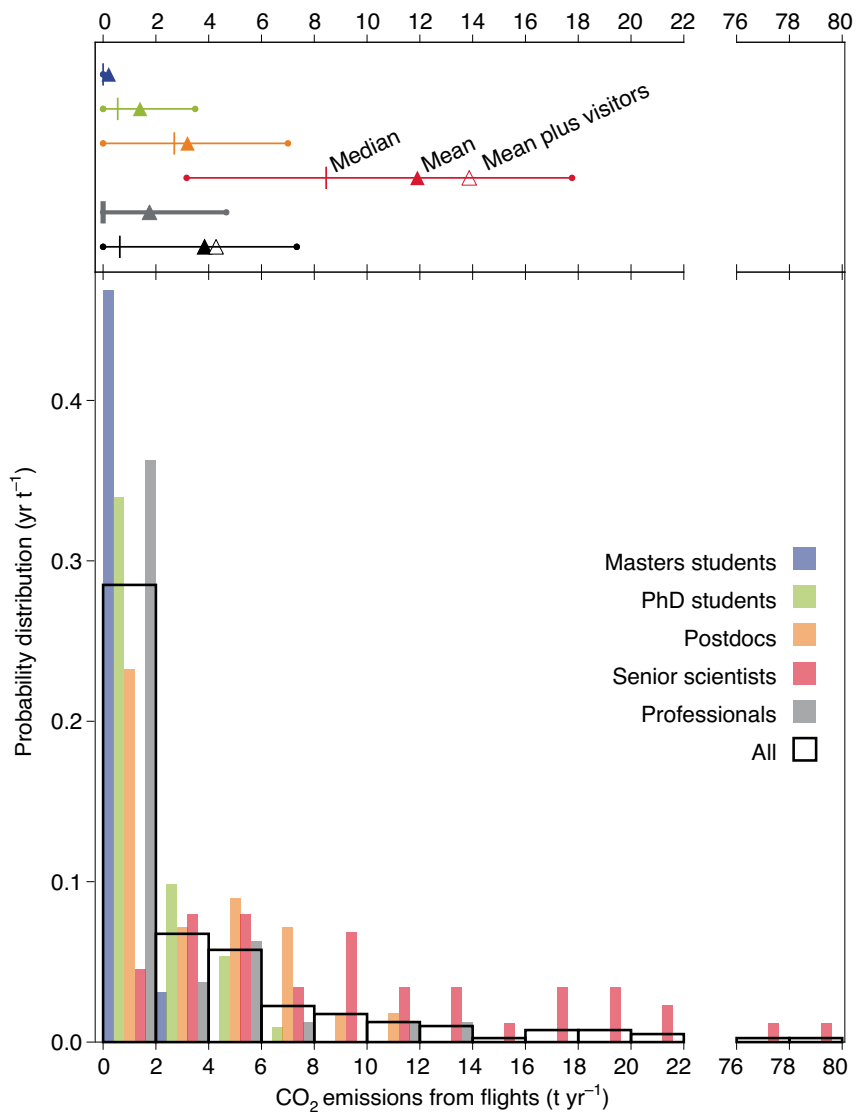

Fig. 1 | Distributions of ICRAR-UWA staff's air travel $\mathrm{CO}_{2}$-equivalent emissions. Bottom panel: normalized histograms of individuals' annual emissions for 2018 and 2019 (two entries per person) from each staff type in bins of width $2 \mathrm{t} \mathrm{yr}^{-1}$; bar thicknesses (except for the 'all' category) have been artificially reduced to visually separate each distribution. Top panel: mean of each distribution (closed triangles, where open triangles add the contribution from visitors), along with their medians (vertical dashes), and 16th and 84th percentiles (dots connected by horizontal bars). These values are provided in Table 2. Figure produced using matplotlib ${ }^{45}$.

to 9.5 tonnes. The average postdoc emits around a third that of an average senior staff member (roughly one international and one domestic trip each year). The average $\mathrm{PhD}$ student emits less than half that of an average postdoc (two to three domestic trips each year, or one international trip every two years).
In total, the flight emissions from ICRAR-UWA staff members over the two-year period was $768 \mathrm{tCO}_{2} \mathrm{e}$. A further $86 \mathrm{tCO}_{2} \mathrm{e}$ came from guest bookings, that is, travel booked by ICRARUWA staff for external visitors and collaborators. It is important that these bookings are not ignored, because if the same study were conducted at those guests' home institutes, those flights would probably not be captured by their systems. Likewise, there could well be other work-related flights that ICRAR-UWA staff members took over this period that were booked externally and are thus not considered here. Incorporating captured guest flights into our figures compensates for this. In all instances, a senior member of staff was the host for the guests, so this reasonably should only contribute towards the figures for senior staff and totals. We include a second column for means in Table 2 that appropriately takes guest flights into account.

Remarkably, the average per-person emissions from flights of PhD students, postdocs and senior scientists combined at ICRAR-UWA is exactly the same as the estimate for CAS (see the

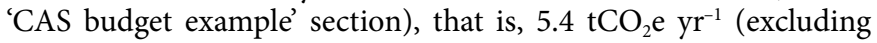
guests' flights). After adding guest bookings, this average increases to just over $6 \mathrm{tCO}_{2} \mathrm{e} \mathrm{yr}^{-1}$.

Despite Perth's relative isolation (it is the second-most isolated major city globally, based on nearest-neighbour distance of cities with populations above one million), the travel budgets of research institutes in Perth do not necessarily exceed that of equivalent institutes elsewhere in Australia. While a domestic trip for those living on the continent's east coast might mean lower emissions (see Table 1), this is probably counterbalanced by an increase in the number of domestic trips. International travel comes at a heavy carbon cost regardless of the Australian city of origin.

National extrapolation. Official figures submitted as part of the 2019/2020 mid-term review of the Australian astronomy decadal plan suggest there are currently 365.2 FTE research staff nationwide, covering academic levels A-E, that is, junior postdocs through to full professors. These figures will be made public as part of the mid-term review process. Consistent with our earlier definition, if we consider postdocs to hold temporary contracts and be employed at either academic level A or B, then postdocs account for 166.2 of those FTEs. That leaves 199 'senior scientists', which we again consider as those at academic level C and above, and/or those with permanent employment. Five additional FTEs fall outside the standard university employment levels, which we do not categorize here. 326.5 FTE astronomy PhD students are enrolled nationwide, as are 72 FTE masters students. An earlier figure from 2014 suggested 242 support staff were also employed across the country ${ }^{22}$, which we equate to our 'professional' category. 
Combining these numbers with the means in Table 2 (including the guest contribution to senior scientists) gives an estimate of the

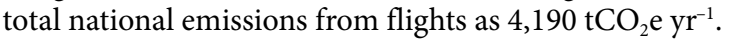

Supercomputer usage. As described in a recent white paper $^{23}$, the estimated computing requirements of Australian astronomers is 400 million CPU core-hours (MCPUh) per annum, expected to rise

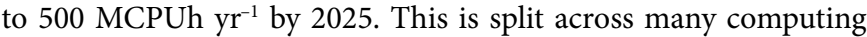
facilities, including both domestic and international supercomputers. Each has its own energy efficiency and is powered by different sources. It is therefore non-trivial to translate this level of computer processing into a rate of $\mathrm{CO}_{2}$-equivalent emissions.

The three most notable supercomputing centres for Australian astronomers are the National Computing Infrastructure (NCI) in the Australian Capital Territory (ACT), the Pawsey Supercomputing Centre in Western Australia (WA), and the OzSTAR supercomputer in Victoria. We contacted each of these to request official figures on the energy/emission requirements that would allow us to estimate astronomers' computing carbon footprint as accurately as possible. Unfortunately, Pawsey was the only centre that responded with data. We therefore extrapolate from these data to estimate the national computing emissions of Australian astronomers.

Figures provided to us privately by Pawsey show that the centre consumed 10.94 GWh of electricity in the 2018/2019 financial year, $<100 \mathrm{MWh}$ of which came from their own solar panels. While one of Pawsey's two solar inverters was down for much of this period, we can reasonably estimate that $99 \%$ of the electricity powering Pawsey comes from the grid. 25\% of Pawsey's computing resources are allocated to astronomy through the dedicated Galaxy supercomputer ${ }^{24}$. We can therefore estimate that Australian astronomers require 2.7 GWh $\mathrm{yr}^{-1}$ of electricity for their Pawsey usage alone (this is probably a lower limit, as other machines at Pawsey-for example, Magnusare used by astronomers too). In southwest WA, electricity currently carries a carbon cost of $0.75 \mathrm{kgCO}_{2} \mathrm{e} \mathrm{kWh}^{-1}$ (we account for both 'scope 2' and 'scope 3' emissions when considering mains power consumption throughout this paper; in principle, this includes the emissions associated with extraction and burning of the fuel used to produce the electricity, as well as losses in transmission $)^{25} .2 .7$ GWh $\mathrm{yr}^{-1}$ at Pawsey therefore translates to $2.0 \mathrm{ktCO}_{2} \mathrm{e} \mathrm{yr}^{-1} .51 .1$ MCPUh were consumed on Galaxy for radio astronomy during the $2018 / 2019$ financial year ${ }^{24}$, implying a carbon cost of $\sim 40 \mathrm{tCO}_{2} \mathrm{e}$ MCPUh $^{-1}$.

Given the above, we estimate the net power required to run code on a supercomputer that includes all overheads and cooling to be $\sim 53 \mathrm{~W}$ per core. In theory, this value could actually be higher for many facilities, as Pawsey uses a groundwater cooling system that should reduce the energy requirements of cooling. Nevertheless, if we assume that $53 \mathrm{~W}$ per core is typical for most supercomputers, then we need only consider where other commonly used facilities are, and the emissions per kWh there. In Victoria and the ACT, electricity emissions are 1.17 and $0.92 \mathrm{kgCO}_{2} \mathrm{e} \mathrm{kWh}^{-1}$, respectively ${ }^{25}$. Despite these being official numbers from the Australian Government, we highlight an important caveat regarding the emissions from electricity use in the ACT later on. For now, we take those numbers at face value. Assuming a ratio of 3:2:1 for NCI:Pawsey:OzSTAR (ACT:WA:Victoria) usage in astronomy (a difficult ratio to gauge with publicly available information), this gives an average of $0.905 \mathrm{kgCO}_{2} \mathrm{e} \mathrm{kWh}^{-1}$ or $\sim 48 \mathrm{tCO}_{2} \mathrm{e} \mathrm{MCPUh}^{-1}$.

It is important to note that only $\sim 60 \%$ of Australian astronomers' supercomputer usage is from domestic facilities ${ }^{23}$. The average emissions per kWh for countries in the Organisation for Economic Co-operation and Development (OECD) is roughly half that of Australia's. Accounting for this-assuming it reflects where the offshore supercomputers that Australian astronomers use arereduces the average emissions for Australian astronomers' supercomputing time to $\sim 38 \mathrm{tCO}_{2} \mathrm{e} \mathrm{MCPUh}^{-1}$.
With all of this in mind, we estimate that the total emissions from

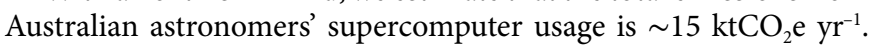
This is nearly quadruple the value from flights (see the 'National extrapolation' section). Dividing across all senior staff, postdocs and $\mathrm{PhD}$ students gives a mean supercomputing carbon footprint of $\sim 22 \mathrm{tCO}_{2} \mathrm{e} \mathrm{yr}^{-1}$ per researcher. Note that we have implicitly assumed that cores on local clusters in Australia carry the same power and carbon requirements as cores on supercomputers; the $400 \mathrm{MCPUh}$ $\mathrm{yr}^{-1}$ figure should include the use of local clusters. Similar to flights, we expect that many of the people being averaged over will require relatively negligible computing time, and thus the mean emissions per researcher will be much less than the actual emissions of the researchers who have a heavy reliance on high-performance computing.

While it is difficult for us to quote an uncertainty on this number within a specified confidence interval, we can take $28 \mathrm{tCO}_{2} \mathrm{e}$ $\mathrm{yr}^{-1}$ per astronomer as a fair upper limit (the figure we would have derived had we not accounted for the lower overseas emissions for electricity). Because of the considerable production of renewable energy that the ACT is responsible for ${ }^{26}$, one can argue that emissions from NCI should be treated as zero. Taking that argument, while maintaining the assumption that $30 \%$ of Australian astronomers' computing is done in the ACT, would lead to a value of $\sim 14$ $\mathrm{tCO}_{2} \mathrm{e} \mathrm{yr}^{-1}$ per researcher. This provides a reasonable estimate of a lower bound.

Observatories and telescopes. Another important source of emissions is the operation of observatories and telescopes. We sought information from several observatories regularly used by Australian astronomers regarding their emissions from operations (for example, power consumption). While the information provided to us is not a complete accounting of all relevant domestic and international observatories (not all places we contacted supplied data), we can place a meaningful lower limit on the total electricity and emissions requirements for Australian astronomers to conduct observations.

In a private communication, the Australia Telescope National Facility (ATNF, part of CSIRO) provided us with the electricity consumption of all observatories they operate over a one-year time frame. The sites considered include the Australia Telescope Compact Array (ATCA), the Parkes Observatory, the Mopra Radio Telescope, and the Murchison Radio-astronomy Observatory (MRO). ATCA, Parkes and Mopra all use mains power (with backup diesel generators) and are all situated in New South Wales (NSW). Those three sites consumed a combined total of 3,760 MWh of electricity over the year ending 29 February 2020, including all the telescopes, buildings and integral facilities on-site. ATCA accounts for 1,920 MWh, with $\sim 70 \%$ of its observing time allocated to Australia-based principal investigators (PIs) in 2018 ${ }^{27}$. Parkes accounts for 1,550 MWh, with $\sim 55 \%$ allocated to Australian PIs in $2018^{27}$. The remaining 290 MWh covers Mopra, although it is harder to obtain a fraction of time spent by Australian PIs on this telescope. An earlier report from $2015^{28}$ shows eight programmes were run on Mopra in the year prior, with three-eighths of the first-name observers identified as belonging to Australian institutions. Given the carbon cost of $0.92 \mathrm{tCO}_{2} \mathrm{e} \mathrm{MWh}^{-1}$ for mains power in $\mathrm{NSW}^{25}$, the combined operation of ATCA, Parkes and Mopra produces $\sim 3.5 \mathrm{ktCO}_{2} \mathrm{e}$ of emissions per year, with a contribution based on Australian astronomers'

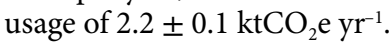

The MRO hosts both the Murchison Widefield Array (MWA) and the Australian Square Kilometre Array Pathfinder (ASKAP). The isolation of the MRO in WA means it is not connected to mains power. Instead it is powered by a combination of on-site solar photovoltaics and diesel. Once operating at maximum capacity, the solar array is expected to cover $>40 \%$ of the site's electricity needs. As of yet, it has not reached this capacity. Over the 2018/2019 financial year, the MWA and ASKAP consumed a total of 4,110 MWh of 
electricity: 3,360 MWh for ASKAP, 520 MWh for the MWA, and $230 \mathrm{MWh}$ from transmission losses. $600 \mathrm{MWh}$ of this came from solar energy, and the rest from diesel. An additional $200 \mathrm{MWh}$ was consumed at the Boolardy accommodation facility, with roughly a third of this estimated to come from solar, and the rest diesel. Based on figures from the Australian Government ${ }^{25}$, the carbon cost of burning diesel for energy is $266 \mathrm{kgCO}_{2} \mathrm{e} \mathrm{MWh}^{-1}$ (this covers 'scope 1 ' and 'scope 3' emissions, that is, the on-site emissions from the burning of diesel and an approximate consideration of indirect emissions associated with its production and transport; the latter is probably an underestimate in the case of the MRO). This implies that the MRO currently produces greenhouse gas emissions at a rate of $\sim 0.95 \mathrm{ktCO}_{2} \mathrm{e} \mathrm{yr}^{-1}$. Based on the facts that $87.5 \%$ of MWA observing time was led by Australia-based PIs in 2019 and $\sim 100 \%$ of current ASKAP operations are Australia-led, we estimate Australian astronomers' contribution to MRO emissions as $\sim 0.93$

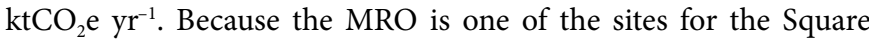
Kilometre Array (SKA), its power consumption is expected to notably increase with time as SKA operations ramp up. An increased fraction in dedicated solar power will help offset any rise in the site's emissions though.

The W. M. Keck Observatory in Hawaii provided us with an estimate of their $\mathrm{CO}_{2}$ emissions from on-site electricity and vehicle use. The latter is only a minor contributor. No flights to or from the observatory were included (flights to the observatory made by Australian astronomers have already been accounted for in the 'Flights' section). The total $\mathrm{CO}_{2}$ emissions reported to us were reduced pro-rata with Australia's current official proportion of Keck observing time of ten observing nights. Noting that Keck operates two near-identical telescopes, there are 730 possible observing nights per (non-leap) year. Given this, an initial estimate of Australia's share of Keck's $\mathrm{CO}_{2}$ emissions is $35 \mathrm{t} \mathrm{yr}^{-1}$. Evidently, this is very small compared to emissions from Australia's use of its own domestic facilities. The Australian astronomical community has had access to up to 40 nights per year on Keck in the past, but even the emissions from that would be almost negligible compared to the sum of ATNF observatories.

We note that the European Southern Observatory (ESO) has already commissioned a study of the emissions of its sites, but the results were pending at the time of writing. Should these be made publicly available, this could prove a useful resource from 2020 onwards. Now a strategic partner of ESO, Australia's emissions contribution to the use of those observatories should be taken into account for completeness.

With the information we have, we can confidently place a lower limit on the observatory-based emissions of Australian astronomers of $3.3 \mathrm{ktCO}_{2} \mathrm{e} \mathrm{yr}^{-1}$. Contributions from the Siding Spring Observatory (which hosts the Anglo-Australian Telescope, the ANU (Australian National University) $2.3 \mathrm{~m}$ telescope, the SkyMapper Telescope, and the UK Schmidt Telescope) and ESO facilities are the most notable exclusions from this estimate. Any involvement that Australian astronomers have in space telescopes has not been accounted for here either.

Campus operations. Office spaces and their machinery also contribute to work-related carbon emissions. While a specific analysis of all the buildings that house astronomy departments in Australia is left for future investigation, we can again use ICRAR-UWA as an example and extrapolate. ICRAR-UWA lies in the Ken and Julie Michael Building at UWA. Figures provided to us by UWA suggest that powering the entire building produces $618,772 \mathrm{kgCO}_{2} \mathrm{e}$ $\mathrm{yr}^{-1}$. ICRAR occupies $48 \%$ of the building's floor area, implying that

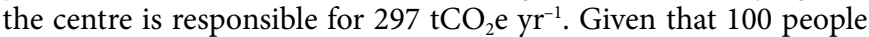
have a desk at ICRAR-UWA (see Table 2), this implies an average of $\sim 3 \mathrm{tCO}_{2} \mathrm{e} \mathrm{yr}^{-1}$ per person for office building requirements. Extrapolating this to the $\sim 1,000$ astronomers and support staff nationwide (see the 'National extrapolation' section) implies total emissions of $\sim 3 \mathrm{ktCO}_{2} \mathrm{e} \mathrm{yr}^{-1}$.

A caveat to the building power requirements of ICRAR-UWA is that this includes powering the Hyades computing cluster. In principle, the emissions from the use of local clusters have already been accounted for in the 'Supercomputer usage' section. However, the entire Hyades system only has 92 cores, meaning it must account for less than 0.8 MCPUh $\mathrm{yr}^{-1}$. Recognizing that ICRAR-UWA makes up $\sim 10 \%$ of the national community, any potential 'double counting' of computing requirements must be less than $2 \%$ of the total from the 'Supercomputer usage' section (that is, $<0.3 \mathrm{ktCO}_{2} \mathrm{e} \mathrm{yr}^{-1}$ ). In reality, local clusters like Hyades almost never operate near their full capacity.

An additional caveat is some of the support staff who are based at observatories might already have their office requirements covered in the 'Observatories and telescopes' section. It might be more appropriate to only extrapolate the per-person office power requirements to $\sim 800$ people. With both caveats, the true office-based emissions of Australian astronomers might be as low as $\sim 2.2 \mathrm{ktCO}_{2} \mathrm{e} \mathrm{yr}^{-1}$.

Summary of emissions. Our findings are that the largest contributor to Australian astronomers' emissions is supercomputing. At $\sim 15$

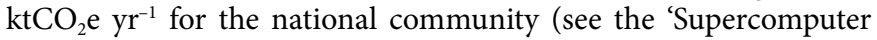
usage' section), this is more than all other sources of work-related emissions combined. This figure is primarily an extrapolation from power usage data we received from a single supercomputing facility (Pawsey). There are many sources of uncertainty contributing to this figure that we have not quantified precisely. With differing assumptions about how emissions from the ACT and non-Australian supercomputers are accounted for, this value could actually be as low as 9.5 or as high as $19 \mathrm{ktCO}_{2} \mathrm{e} \mathrm{yr}^{-1}$.

Despite sometimes garnering the most attention in conversation, flights rank a distant second (at best), totalling $\sim 4.2 \mathrm{ktCO}_{2} \mathrm{e} \mathrm{yr}{ }^{-1}$ (see the 'Flights' section). This figure is largely based on an extrapolation of one institute (ICRAR-UWA), but it is entirely consistent with totals from a second institute (CAS). The formal uncertainty carried through from the jackknifing uncertainties given in Table 2 is effectively negligible, but it does not sufficiently account for potential variation across institutes in the country. At a precision of one significant figure, we can fairly confidently say the value is near to

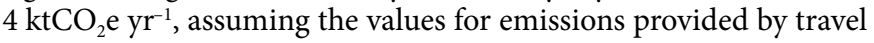
agencies and airlines do not carry systematic uncertainties greater than $\sim 10 \%$ (which we do not know). Based on this, the uncertainty in our figure for flight emissions should be of order a few hundred $\mathrm{tCO}_{2} \mathrm{e} \mathrm{yr}^{-1}$. However, we have not explicitly accounted for the altitude of aeroplane emissions, which is particularly problematic due to the production of contrails ${ }^{29,30}$. In essence, the effective radiative forcing from aeroplane emissions at altitude could be several times that of their nominal $\mathrm{CO}_{2}$ emissions. This systematic error is probably our greatest source of uncertainty.

The powering of observatories ranks third in emissions at $>3.3$

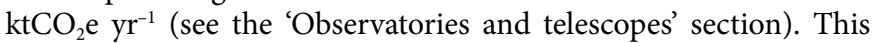
is based on the total power requirements of ATCA, the MRO, the Parkes Observatory and Mopra-accounting for the fraction of Australian PI time on these instruments-with the additional but small contribution from Australia's time on Keck. There are many other observatories that Australian astronomers use, and thus we can only provide a lower limit here. In reality, the emissions from observatory operations could well exceed that of astronomers' flights.

Finally, emissions associated with powering astronomers' office buildings are approximated to be $2.2-3.0 \mathrm{ktCO}_{2}{\mathrm{e} \mathrm{r}^{-1}}$ nationwide (see the 'Campus operations' section). Again, this is based on an extrapolation from ICRAR-UWA, and thus we may have underestimated the true uncertainty.

A visual summary of these four sources of emissions and their estimated uncertainties is provided in Fig. 2. Summed together, 


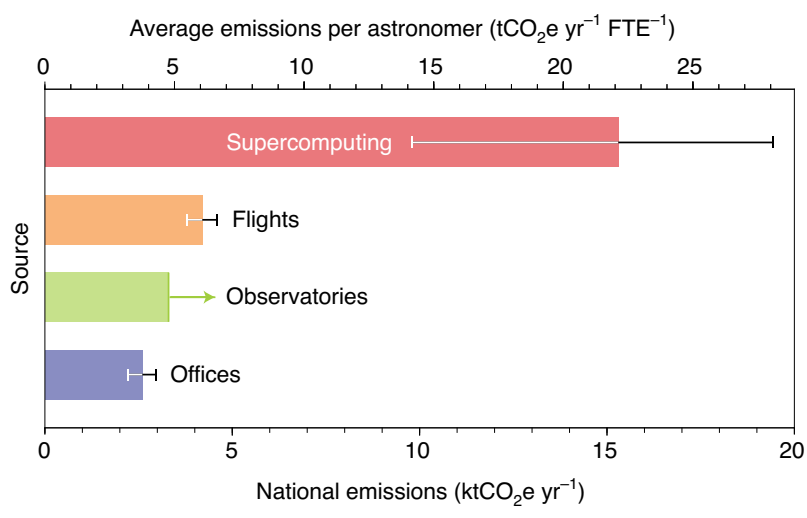

Fig. 2 | Breakdown of the four sources of Australian astronomers' emissions considered in this work. Error bars provide an estimate of our uncertainties, but should not be interpreted as formal confidence intervals. The value for observatories is a lower limit. 'Per astronomer' refers to the 691.7 FTEs including PhD students, postdocs and senior researchers. Values are summarized in the 'Summary of emissions' section. Figure produced using matplotlib ${ }^{45}$.

the Australian astronomy industry is responsible for emitting $\gtrsim 25$ $\mathrm{ktCO}_{2} \mathrm{e}$ of greenhouse gases per year. Dividing this across the combined 691.7 FTE of senior scientists, postdoctoral researchers and $\mathrm{PhD}$ students implies an average of $\gtrsim 37 \mathrm{tCO}_{2} \mathrm{e} \mathrm{yr}^{-1}$ per astronomer. This means the work-based emissions of the average Australian astronomer exceed the combined work-plus-life emissions of the average non-dependant living in Australia by $>40 \%$. Globally, this is around five times the average work-plus-life emissions per non-dependant. Hypothetically, if half of all emissions were associated with people's work (and the other half with their lifestyles), it would follow that an Australian astronomer's job is around three times as carbon-intensive as the average job in Australia, and around ten times that of the average job globally. While there are surely plenty of examples of other jobs that are equally or more carbon-intensive, no such comparison absolves anyone of responsibility.

\section{Solutions and discussion}

To contribute to the mitigation of unsustainable climate change, the astronomy community must focus on reducing the high rate of emissions found in the previous section. In this section, we outline potential strategies to achieve this goal.

Reduce flying. Advances in aeroplane technology have helped to reduce the average emissions per passenger per unit distance in recent years. From 2012 to 2016, the Australian aviation industry saw a $6.8 \%$ increase in fuel efficiency ${ }^{20}$. This was, however, counteracted by a $16.8 \%$ increase in fuel consumption due to a continual rise in airline traffic ${ }^{20}$. While there exist prospects for greater increases in fuel efficiency in future, this is not a domain that astronomers are likely to influence or accelerate. The only practical action that astronomers can take to reduce their flight emissions is to fly less.

The challenge then becomes: without flying, how do we achieve all the same things that up until now have involved air travel? While potentially a confronting question for astronomers used to a high frequency of travel, events in 2020 have already demonstrated that this need not be a daunting challenge any longer.

At the time of finalizing this paper, the outbreak of COVID-19 was declared a pandemic. This has forced many people to cancel travel plans and work remotely. Already, this is precipitating cultural change among astronomers, whereby online meetings have become commonplace. There exists an opportunity here to learn from this situation, enabling the global astronomical community to carry forward a low-travel, technology-focussed approach to communication and collaboration. To achieve this, we need to be deliberate about not defaulting back to our previous travel habits. The suggestions below consider what we can do (and/or should continue to do) once global restrictions on movement are lifted.

Perhaps the simplest and most obvious occasions where flying can be eliminated (or remain absent) are short meetings (one or two days long) with a small number of people $(\lesssim 10)$, including, for example, time-allocation committees and executive committees (Wynes and Donner ${ }^{18}$ call for similar action). In principle, these could easily (continue to) be done via readily available video conferencing software. Despite the often quoted yet anecdotal benefits of people's physical presence at meetings, the justification to fly thousands of kilometres for the sake of a short discussion is tenuous in the era of climate change action.

An additional avenue by which our flight load can remain low is to (further) conduct observations remotely, rather than travelling to observatories. This practice is already being increasingly adopted globally. In Australia, this has been facilitated by the automation of facilities such as the ANU $2.3 \mathrm{~m}$ telescope and Parkes radio telescope. Remote observing stations for the Anglo-Australian Telescope that are in several locations also help in this capacity. By having access to remote observing facilities at each of the major astronomy hubs in the country, not only can flights be reduced, but so too can accompanying financial costs. For larger-scale, international facilities, the Keck remote observing room at Swinburne is open to the Australian astronomical community and provides an alternative to international travel, even if it means domestic travel. Observations conducted via ESO can be done in 'service mode' or 'designated visitor mode', nullifying the need to travel to ESO sites.

Inevitably, it seems conferences must move to a space where virtual attendance is also the norm. Other research fields acknowledge this and have already started experimenting with online conferences (prior to COVID-19-driven social distancing) ${ }^{31}$. To enable this, we must ensure conferences and meetings have adequate video conferencing systems available. This could mean investing in hardware and/or software to meet the requirements of running said meetings smoothly. As a proactive example, the ARC Centre of Excellence for All Sky Astrophysics in 3 Dimensions (ASTRO 3D) is currently considering whether the development of software beyond the capabilities of that regularly used by academics is warranted and worth funding. Members of conference organizing committees should not only have a plan for how they will make remote attendance possible, but also promote and/or advertise this as an option. Indeed, several major astronomy meetings in 2020 will be run (or have already been run) entirely remotely because of COVID-19, including those of the European Astronomical Society and American Astronomical Society, each of which typically attracts of order 1,000 participants. Given that the logistical challenge of running a major conference online far exceeds that of conducting observations or a committee meeting, we should treat these conferences as opportunities to experiment, paying close attention to the aspects that work (that is, lead to a successful meeting, comparable to our experience of in-person conferences) and those that do not.

An emphasis on virtual meetings has the added benefit of increased inclusivity. Removing the need to travel enables those who are limited in their opportunity to travel (be it because of finances, health, carer responsibilities or other reasons) to more readily participate. Even for those without stringent limitations, a reduction in travel alleviates limitations on people's time, and thus increases participation opportunity for everyone.

We suggest that those wishing to travel should have to justify to their travel approvers (1) why alternatives to travel are unsatisfactory, and (2) why their proposed trip is worthy of contributing to climate change. If travel is approved, travellers should take 
careful note of flight options, as the route, airline and aeroplane model can all influence the emissions per passenger of the journey. Fewer flights with lower emissions should be preferred to monetary savings per journey.

On the ECR argument. One reason often cited against flight reduction is that it might harm the careers of early-career researchers (ECRs) and late-stage PhD students. After all, the astronomy job market is incredibly competitive, and the majority of astronomy $\mathrm{PhDs}$ will not find permanent positions in the field ${ }^{32-34}$. A lack of exposure might therefore disadvantage a job applicant, thereby causing them to become one of the many who 'don't make it', despite being more than capable. There are several problems with this argument.

For one, it is entirely anecdotal. To our knowledge, there has not been a systematic study of the career pathways of astronomy $\mathrm{PhDs}$ and whether their frequency of flying in the early stages of their career had any effect on either their decision to stay in the field or their ability to progress had they chosen to stay. One could speculate that a minimal amount of international exposure might be necessary to get one's foot in the door, but the job-hiring and grant-winning processes are stochastic. One could therefore equally speculate that, at some point, the probability of an application being successful as a function of the candidate's exposure might saturate.

The argument also encourages escalation. Competitive people will always look for a way to stand out. If we tell our students and ECRs that they will not stand out if they do not fly to speak at conferences and the like, then not only will they all fly, but the most competitive ones will find an additional means of outdoing their peers (which might mean flying even more). Instead, we should focus on de-escalating the situation. If it is globally mandated that flying should be minimized, then no ECR will be at any disadvantage to their peers by flying less, because everyone will be doing it. In principle, this should have the added positive effect of alleviating some (but certainly not all) anxiety surrounding the overly competitive nature of astronomy, which is one of the frequently cited reasons why people choose to leave the field ${ }^{35,36}$.

It would help to build a culture where values like environmental sustainability are not only supported, but are encouraged and factored into the job-hiring process (for a related discussion, see ref. ${ }^{37}$ ). Senior members of the community hold the greatest power in effecting this culture change. They also have the greatest responsibility to reduce flight emissions, based on the numbers in Table 2, and suffer the least risk in doing so, given that their employment is ongoing. While we suggest that ECRs should reduce their flying, the onus is not necessarily on ECRs in the first instance.

On carbon offsetting. Carbon offsetting is often cited as a method by which one can reduce their net carbon footprint, be it from flying or other sources. In essence, the idea is that by giving money to a scheme that will reduce emissions elsewhere or, ideally, help to remove greenhouse gases from the atmosphere, one offsets the emissions they are personally responsible for. While not devoid of merit, both the principle and practice of carbon offsetting has been widely questioned. Some critics, for example, have likened it more to purchasing absolution of guilt than having a tangible impact on greenhouse gas concentrations in the atmosphere ${ }^{38,39}$.

There are a wide range of offsetting schemes that exist. It is often assumed that offsetting means planting trees, but this is rarely the case. In Australia, airline offsets tend to fund land conservation or fire abatement (see Qantas and Virgin, the country's biggest carriers). While these are worthy causes for investment, their being funded simply prevents potential future emissions (or prevents the reduction of the land's ability to sequester carbon from the atmosphere), and does nothing to remove the greenhouse gases added to the atmosphere from aeroplanes. Even if all offsets were hypothetically funding reforestation, this would not solve climate change in a world where we continue to fly. The solution to reducing the concentration of $\mathrm{CO}_{2}$ in the atmosphere (and the ocean) requires both reforestation and emission reductions ${ }^{38}$.

That said, provided those paying for offset schemes understand that it is not itself a solution, it is better to offset than not. Of course, this does not have to be limited to air travel; if we are to offset our flights, we should also offset our power consumption (and other activities), especially that required for supercomputers, at least in the interim.

It is important to choose and investigate an offset scheme carefully; it does not have to be affiliated with an airline. Each astronomy department should consider a local scheme with tangible benefits to the environment, and ensure a fraction of their budget (travel or otherwise) is allocated for that scheme.

Renewable energy sources. Technology already exists for reducing our carbon footprint from supercomputer usage and other highly electricity-demanding operations. It all comes down to what generates the energy. Much of Australia's power comes from coal burning and other greenhouse gas-emitting sources ${ }^{25}$. This is despite the fact that it has been known for years that it is feasible for Australia to be powered entirely by renewables ${ }^{40,41}$, contrary to the narrative repeated by some of our politicians and other sceptics ${ }^{42}$. Realistically, it will take time for the country to continue its transition to renewables (as it will for the world). We should, therefore, take action ourselves to ensure our electricity-demanding operations are powered by the greatest fraction of renewables possible.

This means we should carefully choose the supercomputers we use, strongly favouring those certified as being powered predominantly by renewables. Concurrently, we should be lobbying and/or helping the facilities we currently use to establish their own renewable energy sources. An obvious first step would be to install solar panels at the facilities where they are not already present. Some efforts in this direction have already been made. For example, the roof of NCI holds 600 'sliver-cell' solar panels, generating $\sim 93.5 \mathrm{~kW}$ of carbon-free electricity. The total power consumption of facilities like NCI is much greater than this though. Dedicated renewable energy farms that cover a much larger area than a building's roof (realistically, off-campus) are ultimately needed. As mentioned in the 'Supercomputer usage' section, Pawsey currently covers $\sim 1 \%$ of its power with on-site solar photovoltaics, and is investigating options to increase its renewables fraction in the foreseeable future.

As alluded to in the 'Supercomputer usage' section, the ACT as a whole is now responsible for generating more renewable energy than the energy it consumes ${ }^{26}$. This power is not exclusively consumed in the territory though; rather, it goes into the grid shared with NSW. For reference, the ACT accounts for less than $2 \%$ of the country's population, and is a factor of $\sim 19$ less populous than NSW. One could argue that the operations of NCI should be considered carbon-neutral because its power consumption has (presumably) been accounted for in the ACT's renewables generation. Equally though, any power drawn from the NSW-plus-ACT grid increases the demand, and the supply that meets this is ultimately still backed by emissions-heavy power sources. That is to say, if the operations of NCI were to cease (or reduce), there would be a measurable reduction in emissions. As such, our default stance has not been to treat astronomers' usage of NCI as carbon-neutral (evidently, the ANU does not treat NCI operations as carbon-neutral either). Nevertheless, initiatives to invest in renewables are precisely what we should be supporting. By extension, it seems favourable to support supercomputing facilities that reside in areas whose local governments are of this philosophy. As per the 'Supercomputer usage' section, were we to assume that NCI is carbon-neutral, our figure for the total emissions of Australian astronomers would drop

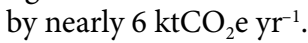


Observatories should also be powered by renewables, which several observatories have already recognized. As mentioned in the 'Observatories and telescopes' section, the MRO has a dedicated hybrid solar-diesel power station, with the potential to supply the site with up to 50\% renewables (although this currently sits at closer to $15 \%)$. ESO's La Silla Observatory has a dedicated solar farm on site too, as will ESO's Extremely Large Telescope.

Many universities in Australia have set targets for approaching carbon neutrality. UWA aims to have its electricity requirements fully covered by renewables by 2025, with plans to further offset other sources of emissions by 2030 . With a slightly more accelerated timeline, the University of Melbourne aims to be energy carbon-neutral by 2021, and fully neutral by 2030. The 2030 goal is also shared by Monash University. Swinburne University plans to procure $100 \%$ renewables by mid-2020, and be carbon-neutral by 2025; perhaps most importantly for astronomers, this will include covering the energy requirements of the OzSTAR supercomputer. The University of Queensland has its own off-site solar farm, which was planned to make the university energy carbon-neutral by 2020 , while the University of New South Wales wants to purchase all its electricity from existing renewables by 2020 . The latest announcement by the ANU states an intent to become 'net negative' in their emissions, although the timescale to achieve this is as yet unclear. Whether the various initiatives of these universities pan out as planned remains to be seen. We can all place pressure on our universities to ensure these policies are carried through or even accelerated where possible.

Create incentives. While the ethical and scientific arguments for significant action to reduce our contribution to climate change undoubtedly have an impact on individuals, the lack of tangible action on this topic thus far suggests that action at an institutional/ governing level is also necessary.

To perhaps state the obvious, creating additional incentives to reduce carbon emissions should, in principle, help to reduce carbon emissions. One option is to establish an award that departments set out to earn. This could be based on purely having low emissions, or could more broadly encompass environmental sustainability. The Astronomical Society of Australia (ASA) went through the same process for a different area of ethical importance several years ago: the Pleiades award for gender equity and diversity. The movement of promoting diversity and equity has resulted in focused committees at most major institutions, an ambassador for women in STEM (science, technology, engineering and mathematics), and numerous national programmes to tackle this problem, including the Science in Australia Gender Equity initiative. We, as a community, should work towards a future where the same importance is placed on our planet as on the people that live on it, to make sure that our legacy is more than just academic. Given the success of the Pleiades and Athena Scientific Women's Academic Network (SWAN) awards ${ }^{43}$, a low-emissions award could be modelled directly on them. The ASA is an ideal organization to lead this because (1) it is a national body and (2) it exists in perpetuity, unlike other national entities (such as Centres of Excellence).

Goal setting. The Paris Agreement lays out goals for emissions reductions on several timescales that will quantifiably limit the rise of the global mean temperature. Loosely, the primary goals are to reduce emissions from 2018 rates by $>50 \%$ by 2030 (or an annual reduction of $7.6 \%$ every year this decade), and $100 \%$ by $2050^{7,44}$. This should keep global heating below $1.5^{\circ} \mathrm{C}$.

One option for the astronomy community is to follow the Paris Agreement percentage targets. However, acknowledging that Australian astronomers' work-related emissions exceed that of the average adult's globally by an order of magnitude (see the 'Summary of emissions' section), our percentage goals should arguably be even bigger; practically, those who emit more have greater potential to reduce emissions, not just absolutely, but also fractionally. A plan for all supercomputers, observatories and offices that Australian astronomers rely on to be powered entirely by renewables would already see a $\sim 90 \%$ reduction in the community's emissions. It is not unfathomable that this could be achieved by 2030, especially as many Australian universities' carbon-neutral plans are already in motion (see the 'Renewable energy sources' section). These will help cut up to $45 \%$ of astronomers' emissions, based on the sum of those associated with offices, NCI, and OzSTAR shown in the 'Sources of astronomers' emissions' section (this fraction will be notably less if ACT emissions were already to be treated as zero). With active commitment moving forward, the community is well positioned to make a real contribution to limiting the effects of climate change. Let's do it!

\section{Ethics statement}

The use of ICRAR-UWA travel data (see the 'ICRAR-UWA travel records' section) was approved for this study via an ethics exemption from the Human Ethics office at The University of Western Australia. Although the data are anonymized in this study, specific informed consent was obtained from anyone deemed potentially identifiable. Appropriate permission from the Centre for Astrophysics and Supercomputing at Swinburne University of Technology was obtained for the use of information presented in the 'CAS budget example' section.

\section{Data availability}

Travel records for ICRAR-UWA staff and students and ATNF electricity data are private. Queries about how the former were processed should be directed to A.R.H.S. Similarly, flight records of CAS and data from Keck are private, but queries regarding these can be directed to M.T.M. Power meter data from Pawsey are also private; requests for these data should be directed to Pawsey themselves, and we would encourage copying in P.J.E. The demographics of Australian astronomers will be made publicly available online with an accompanying white paper; none of the authors are involved.

Received: 27 March 2020; Accepted: 2 July 2020;

Published online: 10 September 2020

\section{References}

1. Ripple, W. J., Wolf, C., Newsome, T. M., Barnard, P. \& Moomaw, W. R. World scientists' warning of a climate emergency. BioScience 70, 8-12 (2019).

2. Friedlingstein, P. et al. Global carbon budget 2019. Earth Syst. Sci. Data 11, 1783-1838 (2019).

3. Allen, M. R. et al. in Global Warming of $1.5^{\circ} \mathrm{C}$, IPCC Special Report (eds Masson-Delmotte, V. P. et al.) 51-92 (IPCC, 2018).

4. Matthews, H. D. \& Caldeira, K. Stabilizing climate requires near-zero emissions. Geophys. Res. Lett. 35, L04705 (2008).

5. Allen, $\mathrm{M}$. R. et al. A solution to the misrepresentations of $\mathrm{CO}_{2}$-equivalent emissions of short-lived climate pollutants under ambitious mitigation. $n p j$ Clim. Atmos. Sci. 1, 16 (2018).

6. Allen, M. R. et al. in Global Warming of $1.5^{\circ} \mathrm{C}$, IPCC Special Report (eds Masson-Delmotte, V. P. et al.) 68 (IPCC, 2018).

7. Rogelj, J. et al. in Global Warming of $1.5^{\circ} \mathrm{C}$, IPCC Special Report (eds Masson-Delmotte, V. P. et al.) 95-174 (IPCC, 2018).

8. De Paolo, D. J. Sustainable carbon emissions: The geologic perspective. Energy Sustain. 2, E9 (2015).

9. Archer, D. \& Brovkin, V. The millennial atmospheric lifetime of anthropogenic $\mathrm{CO}_{2}$. Clim. Change 90, 283-297 (2008).

10. Solomon, S., Plattner, G.-K., Knutti, R. \& Friedlingstein, P. Irreversible climate change due to carbon dioxide emissions. Proc. Natl. Acad. Sci. USA 106, 1704-1709 (2009).

11. IPCC in Climate Change 2013: The Physical Science Basis (eds Stocker, T. F. et al.) Technical Summary (Cambridge Univ. Press, 2013).

12. Cohen, S. States of Denial: Knowing about Atrocities and Suffering (Polity Press, 2000).

13. Walker, I. \& Leviston Z. There are three types of climate change denier, and most of us are at least one. The Conversation https://go.nature.com/2PsYDtI (2019). 
14. Quarterly Update of Australia's National Greenhouse Gas Inventory for March 2019 (Australian Government Department of the Environment and Energy, 2019); https://go.nature.com/30zXG9v

15. Ritchie, H. \& Roser, M. $\mathrm{CO}_{2}$ and Greenhouse Gas Emissions (Our World in Data, 2018); https://go.nature.com/3iO9QSv

16. Matzner, C. D. et al. Astronomy in a Low-Carbon Future (Zenodo, 2019); https://doi.org/10.5281/zenodo.3758549

17. Williamson, K., Rector, T. A. \& Lowenthal J. Embedding climate change engagement in astronomy education and research. Preprint at https://arxiv. org/abs/1907.08043 (2019).

18. Wynes, S. \& Donner, S. D. Addressing Greenhouse Gas Emissions from Business-Related Air Travel at Public Institutions: A Case Study of the University of British Columbia (Pacific Institute for Climate Solutions, 2018).

19. Middleton, C. Environmental sustainability goals drive changes in conference practices. Phys. Today 72, 29 (2019).

20. Managing the Carbon Footprint of Australian Aviation (Australian Government Department of Infrastructure and Regional Development, 2017); https://go.nature.com/3ifQHZn

21. Stohl, A. The travel-related carbon dioxide emissions of atmospheric researchers. Atmos. Chem. Phys. 8, 6499-6504 (2008).

22. Maddison, S. et al. Demographic Survey of Australian Astronomy, Report to the National Committee for Astronomy for the Australian Astronomy Decadal Plan 2016-2025 (2015); https://go.nature.com/30zbJME

23. O'Toole, S., Pindor, B. \& Power, C. Data and Computing White Paper, Decadal Plan for Australian Astronomy Mid-term Review (2019); https://go.nature. $\mathrm{com} / 2 \mathrm{XxSYai}$

24. Annual Report 2018-19: Growing Australia's Scientific Ambitions by Expanding Our HPC Infrastructure (Pawsey Supercomputing Centre, 2018); https://go. nature.com/3fx4B7K

25. National Greenhouse Accounts Factors - July 2018 (Australian Government Department of the Environment and Energy, 2018); https://go.nature. $\mathrm{com} / 2 \mathrm{XxwExK}$

26. Cass, D. Class ACT: How the Australian Capital Territory Became a Global Energy Leader (The Australia Institute, 2019); https://go.nature.com/3fzrpnh

27. CSIRO Australia Telescope National Facility: Annual Report 2018-19 (CSIRO Australia Telescope National Facility, 2019); https://go.nature.com/2Px2V3y

28. CSIRO Australia Telescope National Facility: Annual Report 2015 (CSIRO Australia Telescope National Facility, 2015); https://go.nature.com/3gzPsUd

29. Frömming, C. et al. Aviation-induced radiative forcing and surface temperature change in dependency of the emission altitude. J. Geophys. Res. 117, D19104 (2012)

30. Rädel, G. \& Shine, K. P. Radiative forcing by persistent contrails and its dependence on cruise altitudes. J. Geophys. Res. 113, D07105 (2008).

31. Abbott, A. Low-carbon, virtual science conference tries to recreate social buzz. Nature 577, 13 (2019).

32. Forbes, D. A. So you want to be a professional astronomer! Mercury 37, 24-28 (2008).

33. Metcalfe, T. S. The production rate and employment of Ph.D. astronomers. Publ. Astron. Soc. Pac. 120, 229-234 (2008).

34. Cooray, A., Abate, A., Häußler, B., Trump, J. R. \& Williams, C. C. Astronomy job crisis. Preprint at https://arxiv.org/abs/1512.02223 (2015).

35. Arka, I. The psychological aspects of a well-known issue. Nat. Astron. 2, 16-19 (2018).

36. Karouzos, M. Careers beyond academia. Nat. Astron. 2, 20-23 (2018).

37. Walkowicz, L. M. Choose your own adventure: developing a values-oriented framework for your career. Preprint at https://arxiv.org/abs/1805.09963 (2018).
38. Hansen, J. Storms of My Grandchildren: The Truth about the Coming Climate Catastrophe and our Last Chance to Save Humanity (Bloomsbury Publishing, 2011).

39. Hodgkinson, D. \& Johnston, R. DIY climate action might make us feel good, but it won't solve the problem. The Conversation https://go.nature. com/3kwhCSK (2015)

40. Elliston, B., MacGill, I. \& Diesendorf, M. Comparing least cost scenarios for $100 \%$ renewable electricity with low emission fossil fuel scenarios in the Australian National Electricity Market. Renew. Energy 66, 196-204 (2014).

41. Elliston, B., Riesz, J. \& MacGill, I. What cost for more renewables? The incremental cost of renewable generation - An Australian National Electricity Market case study. Renew. Energy 95, 127-139 (2016).

42. Diesendorf, M. \& Elliston, B. The feasibility of $100 \%$ renewable electricity systems: a response to critics. Renew. Sustain. Energy Rev. 93, 318-330 (2018)

43. Kewley, L. J. Diversity and inclusion in Australian astronomy. Nat. Astron. 3, 1067-1074 (2019).

44. Emissions Gap Report 2019: Executive Summary (United Nations Environment Programme, 2019)

45. Hunter, J. D. Matplotlib: a 2D graphics environment. Comput. Sci. Eng. 9, 90-95 (2007).

\section{Acknowledgements}

S.B., P.J.E. and M.T.M. are supported by the Australian Research Council through project numbers DP180103740, CE170100013 and FT180100194, respectively. This work is an update from a white paper that was commissioned as part of the mid-term review of the 2016-2025 decadal plan for astronomy in Australia. A.R.H.S. thanks L. Staveley-Smith and C. Trott in their capacity as members of the mid-term review panel for their encouragement to write the paper. A.R.H.S. and S.B. thank R. Sharma for extended help in obtaining data pertinent to calculating ICRAR-UWA's emissions. P.J.E. thanks B. Evans and the Pawsey Supercomputing Centre for generously supplying data used in this work. A.R.H.S. thanks P. Mirtschin and P. Edwards at CSIRO for volunteering and providing information on ATNF facilities.

\section{Author contributions}

A.R.H.S. led the writing, analysis and design of this work, but all authors made important contributions to all aspects of this paper. S.B. and A.R.H.S. facilitated the collection of data from ICRAR and produced the figures. P.J.E. liaised with Pawsey to obtain the supercomputing data. M.T.M. provided data for CAS and obtained data from Keck. All authors engaged with observatories' representatives to obtain information.

\section{Competing interests}

The authors declare no competing interests.

\section{Additional information}

Correspondence should be addressed to A.R.H.S.

Peer review information Nature Astronomy thanks Christopher Matzner, Travis Rector and the other, anonymous, reviewer(s) for their contribution to the peer review of this work.

Reprints and permissions information is available at www.nature.com/reprints.

Publisher's note Springer Nature remains neutral with regard to jurisdictional claims in published maps and institutional affiliations.

(C) Springer Nature Limited 2020 